\title{
Investigation of the Dynamical Behavior of a High-Power Laser Diode Subject to Stimulated Brillouin Scattering Optical Feedback
}

Correa-Mena, Ana Gabriela; Lee, Min Won; Zaldvar-Huerta , Ignacio Enrique; Hong, Yanhua; Boudrioua, Azzedine

\section{IEEE Journal of Quantum Electronics}

DOI:

10.1109/JQE.2019.2951110

Published: 01/02/2020

Peer reviewed version

Cyswllt i'r cyhoeddiad / Link to publication

Dyfyniad o'r fersiwn a gyhoeddwyd / Citation for published version (APA):

Correa-Mena, A. G., Lee, M. W., Zaldvar-Huerta , I. E., Hong, Y., \& Boudrioua, A. (2020). Investigation of the Dynamical Behavior of a High-Power Laser Diode Subject to Stimulated Brillouin Scattering Optical Feedback. IEEE Journal of Quantum Electronics, 56(1), [2000406]. https://doi.org/10.1109/JQE.2019.2951110

\section{Hawliau Cyffredinol / General rights}

Copyright and moral rights for the publications made accessible in the public portal are retained by the authors and/or other copyright owners and it is a condition of accessing publications that users recognise and abide by the legal requirements associated with these rights.

- Users may download and print one copy of any publication from the public portal for the purpose of private study or research.

- You may not further distribute the material or use it for any profit-making activity or commercial gain

- You may freely distribute the URL identifying the publication in the public portal ?

Take down policy

If you believe that this document breaches copyright please contact us providing details, and we will remove access to the work immediately and investigate your claim. 


\title{
Investigation of the dynamical behaviour of a high-power laser diode subject to stimulated Brillouin scattering optical feedback
}

\author{
Ana Gabriela Correa-Mena, Min Won Lee, Ignacio Enrique Zaldívar-Huerta Member, IEEE,, Yanhua Hong,
}

\author{
Azzedine Boudrioua
}

\begin{abstract}
In this work, we propose a new scheme of optical feedback using stimulated Brillouin scattering (SBS). A highpower laser diode emitting at $1450 \mathrm{~nm}$ stimulates Brillouin backscattering in a $4 \mathrm{~km}$-long optical fibre and the back-scattered light is injected back into the laser diode for optical feedback. The experimental results with RF spectrum exhibit clearly the Brillouin frequency shift at $11.46 \mathrm{GHz}$. Although the frequency shift is very large, the laser diode subject to optical feedback using SBS shows abundant dynamics. RF spectrum maps are also established with respect to the laser drive current. The results are compared with the ones for conventional optical feedback and it is clearly shown that the dynamical behaviours for both the configurations are very different. The investigation with autocorrelation functions reveals that the time-delay signature vanishes completely using the SBS feedback scheme unlike the conventional feedback one.
\end{abstract}

Index Terms-optical feedback, stimulated Brillouin scattering, chaotic laser

\section{INTRODUCTION}

Optical feedback influence on properties of semiconductor lasers has been studied extensively since the early laser development in the 1970's. The main laser diode properties to be modified when subject to optical feedback are the threshold gain, the resonant frequency and the spectral linewidth[1], [2]. The dynamical behaviour of laser diodes shows different dynamical regimes, including chaotic regime[3]. Optical feedback can be achieved with a simple external mirror[4], [5]. There exist other configurations such as very-short caviry feedback[6], filtered optical feedback[7] and phase-conjugated optical feedback[8], [9]. Thanks to their high-complex dynamical behaviour, chaotic lasers have been used for number of applications such as chaos secure communications[10], [11], [12], chaotic lidar, chaotic radar[13], chaotic optical timedomain reflectometry[14], random number generation[15], reservoir computing[16], [17] and rogue wave generation[18], [19].

Recently, chaotic lasers have been used in the Brillouin scattering area such as suppression of stimulated Brillouin

A. G. Correa-Mena and I. E. Zaldívar-Huerta are with the Departamento de Electrónica, Instituto Nacional de Astrofísica, Óptica y Electrónica, 72000 Puebla, Mexico e-mail: min.lee@univ-paris13.fr

M. W. Lee and A. Boudrioua are with the Laboratoire de Physique des Lasers CNRS UMR 7538, Université Paris 13, Sorbonne Paris Cité, 93430 Villetaneuse, France.

Y. Hong is with the School of Electronic Engineering, Bangor University, Bangor, Gwynedd LL57 1UT, Wales, U.K.

Manuscript received ; revised scattering (SBS) [20], Brillouin optical correlation-domain reflectometry and analysis [21] and dynamic Brillouin grating generation [22], [23]. SBS is an inelastic nonlinear scattering that occurs due to the interaction between the pump and Stokes fields through an acoustic wave. The scattered light is downshifted in frequency because of the Brillouin grating. In a standard single-mode telecom optical fibre, Brillouin frequency shift (BFS) is typically around $11 \mathrm{GHz}$ at $1550 \mathrm{~nm}$ [24].

One of the drawback of optical feedback laser diodes is the time-delay signature (TDS) due to the external cavity round-trip time. The TDS is an important key in secure chaos communications or in random-number generation. In order to enhance the security or the randomness of number generation, the TDS must be suppressed from chaotic signals. Some studies have shown that the TDS can be suppressed using post-signal processing such as self-interference[25], SBS [26], [27] or optical time-lens [28]. However, there is no optical feedback configuration to generate directly TDS-free signals from a laser diode.

In this letter, we propose a new optical feedback configuration using SBS in order to supress the TDS. As the Brillouin back-scattered light frequency is down-shifted by around $11 \mathrm{GHz}$, this configuration can be considered as frequencyshifted optical feedback configuration. Such a configuration has been studied mostly using an acousto-optic modulator[29], [30], never using BFS. Our new scheme also exploits random feedback from SBS which is considered as a random process. The random feedback is new and has never been reported. A high power laser diode is used for stimulating Brillouin scattering in a $4 \mathrm{~km}$-long fibre. Although chaotic behaviour of a laser diode reduces SBS significantly[20], the laser diode shows different dynamical behaviours by injecting back the stimulated Brillouin scattered light into the laser diode. The results will be compared with the ones in a conventional optical feedback (COF) in terms of L-I curves and radiofrequency (RF) spectra. A comparison will also be made using RF spectrum maps with respect to the laser drive current. Moreover, SBS occurs all along the fibre, rather than a distance-defined reflection. Hence, the optical feedback using SBS can be considered optical feedback using an infinite number of external mirrors. This feature gives a possibility of TDS suppression. For this purpose, we will also investigate autocorrelation functions. 


\section{EXPERIMENTAL SETUP}

The schematic diagrams for conventional- and stimulated Brillouin back-scattering- optical feedback are illustrated in Fig. 1(a) and (b), respectively. It consists of a high-power laser diode (LD) of $3.1 \mathrm{~mm}$-long Fabry-Perot cavity, emitting at $1450 \mathrm{~nm}$ (3SP technologies). Such a laser diode is used to pump Raman fibres for Raman amplification. The laser temperature is set to $25^{\circ} \mathrm{C}$ and the maximum free-running laser power is $270 \mathrm{~mW}$ at a current of $1000 \mathrm{~mA}$. The LD light is injected into a $4 \mathrm{~km}$-long single-mode fibre spool through two optical circulators (OC) and a coupler 90/10. The 90\%port of the coupler is used for injecting into the fibre and the power injected into the fibre is reduced to $140 \mathrm{~mW}$ at $1000 \mathrm{~mA}$ because of optical losses from the components. The other $10 \%$-port is used for monitoring the laser output with a $12 \mathrm{GHz}$ photodetector (PD). In order to protect the PD, the monitoring power is attenuated to $1 \mathrm{~mW}$ at the maximum laser power through a variable optical attenuator (VOA). The PD output signal is visualised by a radio frequency (RF) spectrum analyser $(26.5 \mathrm{GHz})$. The optical attenuation of the fibre spool at $1450 \mathrm{~nm}$ is around $1.2 \mathrm{~dB}$ (a linear attenuation of $0.3 \mathrm{~dB} / \mathrm{km}$ ). The isolator placed after the long fibre ensures unidirection, avoiding reflection from the fibre tip.

For COF, the isolator output is injected into the LD via VOA2 to adjust the optical feedback ratio, implementing a conventional closed-loop as depicted in Fig. 1(a). In this work, the feedback ratio is fixed to $0.1 \%(-30 \mathrm{~dB})$ and the round-trip time of the closed loop with the $4 \mathrm{~km}$ long fibre is around $21 \mu \mathrm{s}$. It is also noted that it is incoherent optical feedback. According to Ref. [3] the distance of the optical feedback in semiconductor lasers does not affect Regime IV (chaotic regime). For SBS optical feedback (SBSOF), the back-scattered light of SBS occurred in the fibre is collected by Port 3 of OC 2 and injected into the LD through Port 1 of OC1, setting up a SBS closed-loop configuration as shown in Fig. 1(b). To begin with, the optical feedback ratio with SBS is undefined as the back-scattering rate is unknown. When Port 1 of the OC1 is not connected, the setup is in an openloop configuration.

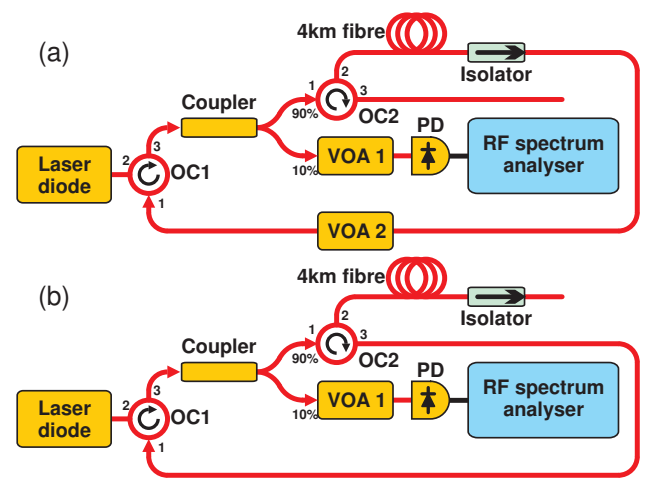

Fig. 1. Schematics of the experimental setup for (a) COF and (b) SBSOF. OC: optical circulator, VOA: variable optical attenuators, PD: photo-detector.

Hence, in this work, three configurations are considered:

1) Open-loop configuration: a free-running laser diode
2) COF configuration: $0.1 \%$ feedback in a ring cavity with a $4 \mathrm{~km}$-long fibre

3) SBSOF configuration: back-scattering cavity with unknown ratio

\section{EXPERIMENTAL RESULTS}

First, L-I characteristics have been investigated in the openloop, COF and SBS closed-loop configurations. Figure 2(a) traces L-I curves measured at the $10 \%$-port of the coupler in the three configurations without VOA1 and PD. In the openloop configuration, the laser threshold is measured as $155 \mathrm{~mA}$ (see the inset of the figure) and the power at $1000 \mathrm{~mA}$ is obtained as $19.6 \mathrm{~mW}$ from the L-I curve shown in the red curve of the figure (lowest curve). Note that the direct laser output power is measured as $270 \mathrm{~mW}$, hence a factor of 14.2 must be taken into account to work out the real laser output power in the figure. The power measured just before injecting into the fibre spool is $140 \mathrm{~mW}$ at $1000 \mathrm{~mA}$ because of the components insertion losses. Therefore, a factor of 7.3 must be considered on the red curve to work out the real injection power. In the COF configuration, the laser threshold is reduced to $133 \mathrm{~mA}$ and the laser output power is increased to $20.6 \mathrm{~mW}$ at $1000 \mathrm{~mA}$ (green curve, uppermost curve). In the SBSOF configuration, the threshold is reduced to $147 \mathrm{~mA}$ as the blue curve (middle curve) indicates, but it is noticed that after 350 $\mathrm{mA}$ the laser output power in slightly increased. So the blue curve starts initially close to the red one, but goes close to the green one after $350 \mathrm{~mA}$. The power is measured as $20.3 \mathrm{~mW}$ at $1000 \mathrm{~mA}$ in this case.

Figure 2(b) plots the power back-scattered from the $4 \mathrm{~km}$ long fibre, which is measured at port 3 of OC2. In the open-loop configuration, some irregular spikes are observed in the red curve. In fact, the free-running laser shows a broad spectrum as shown in Fig. 3(a) and its spectrum is very unstable. Theses spikes are suggested to be intermittent SBS due to this spectrum instability. The power of these spikes can go up to $20 \mathrm{~mW}$. It is found that the base curve underneath spikes is not flat. Actually, the base curve appears like an L-I curve, measuring around $30 \mu \mathrm{W}$ at $1000 \mathrm{~mA}$. This backscattered light is thought to be from Rayleigh scattering with a rate of $-36.7 \mathrm{~dB}$ compared to the injection power. In the COF configuration, the back-scattered light is measured as only $32 \mu \mathrm{W}$ at $1000 \mathrm{~mA}$ and the curve is similar to L-I curve as the green curve (lowest curve) manifests. As the laser outputs chaotic signal in this configuration, it is expected that no SBS is present[20] and the back-scattered light shown in the figure is thought to be as a result of Rayleigh scattering. The scattering rate is measured as $-36.7 \mathrm{~dB}$ which is similar to the one in the open-loop configuration. In the SBSOF configuration, the back-scattered light is measured with another $90 / 10$ coupler. Since the $10 \%$-port is used for monitoring the back-scattered light power, the measured power represented in the blue curve of Fig. 2(b) has been multiplied by a factor of 10. As seen in the figure, the light increases significantly at around $306 \mathrm{~mA}$ unlike the other configurations. It is suggested that this current is the Brillouin threshold current for our setup. At this current, the laser output power in the red curve of 
Fig. 2(a) is obtained as $3.77 \mathrm{~mW}$. By multiplying the factor of 7.3, the power injected into the fibre is expected to be $27.5 \mathrm{~mW}$ which is considered as the Brillouin threshold power. It is clearly seen in the figure that the back-scattered power fluctuates strongly. As will be discussed later, SBSOF causes noise-like intensity fluctuations. In Fig. 2(b), the SBS light saturates to $450 \mu \mathrm{W}$ as increasing the current. Therefore, SBSOF ratio is around $-25 \mathrm{~dB}$ compared to the injection power. The reason for this saturation might come from the fact that as increasing the current, the noise-like fluctuation due to SBSOF becomes stronger, leading to preventing further SBS. Hence, the saturation power of $450 \mu \mathrm{W}$ may be a tradeoff between SBS and noise-like fluctuations in our setup. It is also noticed that the SBSOF ratio is $11.7 \mathrm{~dB}$ greater than that of the Rayleigh scattering rate. In summary, the parameters
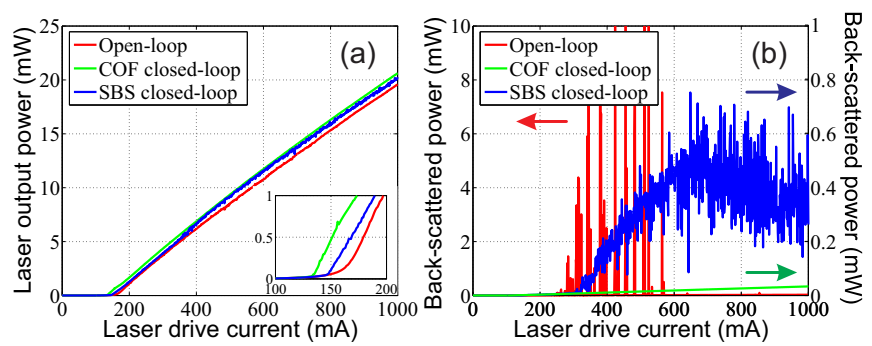

Fig. 2. (a) L-I curves measured at the $10 \%$-port of the coupler and (b) backscattered power measured at port 3 of OC2. See the left y-axis scale for the red curve and the right one for the green and blue curves as indicated with arrows. The inset of (a) is a zoom-in of the L-I curves near the thresholds.

measured in the three configuration are shown in Tab.I:

TABLE I

PARAMETERS MEASURED IN THE THREE CONFIGURATIONS.

\begin{tabular}{|c|c|c|c|}
\hline Configuration & $\begin{array}{l}\text { feedback } \\
\text { ratio }(\mathrm{dB})\end{array}$ & $\begin{array}{l}\text { threshold } \\
\text { current }(\mathrm{mA})\end{array}$ & $\begin{array}{l}\text { Output power }(\mathrm{mW}) \\
\text { at 1000 mA }\end{array}$ \\
\hline Open-loop & N/A & 155 & 19.6 \\
\hline COF & -30 & 133 & 20.6 \\
\hline SBSOF & -25 & 147 & 20.3 \\
\hline
\end{tabular}

The reason for which only three configurations are considered is that these configurations are the most pertinent configurations in our work. The open-loop configuration is the case of a free-running laser. It is important to compare the results without and with feedback. The COF configuration is considered as a reference configuration in our work. In order to compare our new feedback configuration with the $\mathrm{COF}$ configuration, a feedback ratio of $-30 \mathrm{~dB}$ has been chosen in the COF configuration because the feedback ratio from the SBS is around $-25 \mathrm{~dB}$ at most.

Laser output spectra have also been measured in the three configurations. The measurements have been undertaken at the $10 \%$-port of the coupler and the resolution of our optical spectrum analyser is $0.07 \mathrm{~nm}$. The spectrum of the freerunning laser is depicted in Fig. 3(a). It is broad and unstable. Actually, such a laser is used as a pump laser with a fibre Bragg grating for Raman amplifiers. The fibre Bragg grating acts as a filter and mirror for optical feedback, and allows stabilising the optical spectrum. In our setup, no fibre Bragg grating is used and the spectrum is unstable with noise.
Nevertheless, SBS occurs intermittently as seen in Fig. 2(b). In the COF configuration, the laser spectrum changes radically to multimode spectrum as shown in Fig. 3(b). The spectrum is also stabilised without noise in the COF configuration. Figure 3(c) displays the spectrum of the SBSOF configuration and it is different to the spectra in Fig. 3(a) and (b). Although the spectrum of the free-running laser diode is broad and multimode emission-like, SBS still occurs with a small amount compared to the injected power according to the blue curve in Fig. 2(b). The spectrum appears very noisy because in the SBSOF case, the SBS stokes wave with BFS are added into the spectrum for each noise peak, leading to noisier spectrum.

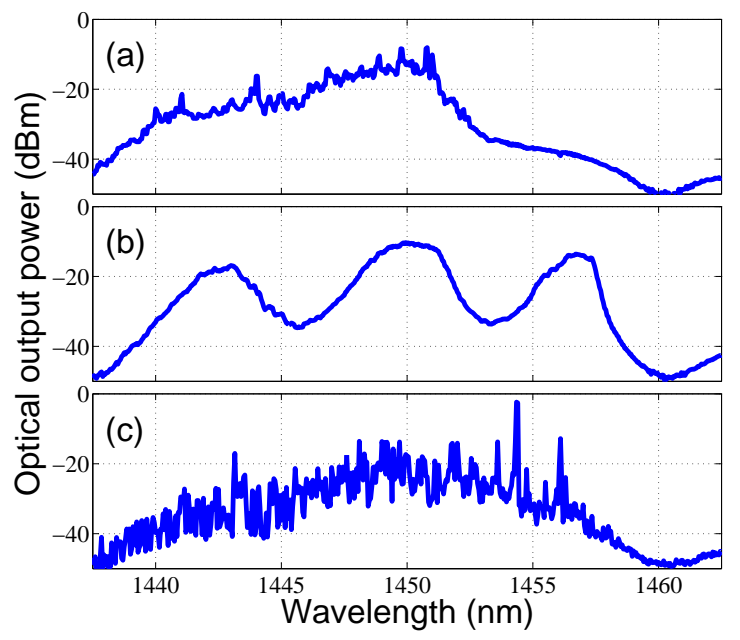

Fig. 3. Optial spectra measured at $1000 \mathrm{~mA}$ for (a) the open-loop configuration, (b) the COF closed-loop configuration with a feedback ratio of $-30 \mathrm{~dB}$ and (c) the SBS closed-loop configurations.

In order to investigate the dynamical behaviour of the laser diode, we have measured RF spectra for the three configurations. Figure 4 depicts spectra measured at 1000 $\mathrm{mA}$ with a video bandwidth of $10 \mathrm{kHz}$ in the SBS, COF closed-loop and open-loop configurations. In the open-loop configuration, the spectrum is almost the same as the noise floor of the system. But a peak at $14.41 \mathrm{GHz}$ is observed in the spectrum. This peak appears to be the internal cavity frequency (side-mode) from the $3.1 \mathrm{~mm}$-long cavity of the LD. Despite of a small feedback ratio of $-30 \mathrm{~dB}$, the spectrum in the COF closed-loop configuration exhibits rich dynamics up to $16 \mathrm{GHz}$ compared to the open-loop one. The peak at around $3 \mathrm{GHz}$ results from the relaxation oscillation frequency of the laser diode. The side-mode at $14.41 \mathrm{GHz}$ is also present. Figure 4(c) exhibits zoom-in spectra at $3 \mathrm{GHz}$. The red trace represents the spectrum of the open-loop noise floor. The blue trace indicates the spectrum for COF. The external cavity mode is clearly seen in the spectrum and the frequency is measured as $47 \mathrm{kHz}$ due to the $4 \mathrm{~km}$-long fibre. Such external cavity frequencies are inevitably present in all feedback configurations. They are also clearly visible even in a very-short cavity of which the frequency is greater than the laser relaxation oscillation frequency[6]. On the other hand, the spectrum in the SBS closed-loop configuration appears very different to that in the COF one as shown in Fig. 4(b). 
The spectrum manifests abundant dynamics in low and high frequency bands and appears noisy. As the optical spectrum in the SBSOF configuration (See Fig. 3(c)) is noisy due to BFS at each noisy peak, the RF spectrum also appears noisy. In Fig. 4(d), the blue trace corresponds to the spectrum for SBSOF and no external cavity frequencies are observed unlike that in the COF configuration. This result is promising for the TDS suppression. It is also seen from the figure that the BFS is present at $11.46 \mathrm{GHz}$ which corresponds to the one calculated with the theory. This is a clear evidence of the presence of SBS in the system. The Brillouin gain spectrum linewidth is measured as $30 \mathrm{MHz}$. It is also clearly noticed that the sidemode is shifted from $14.41 \mathrm{GHz}$ to $15 \mathrm{GHz}$ because of SBSOF.
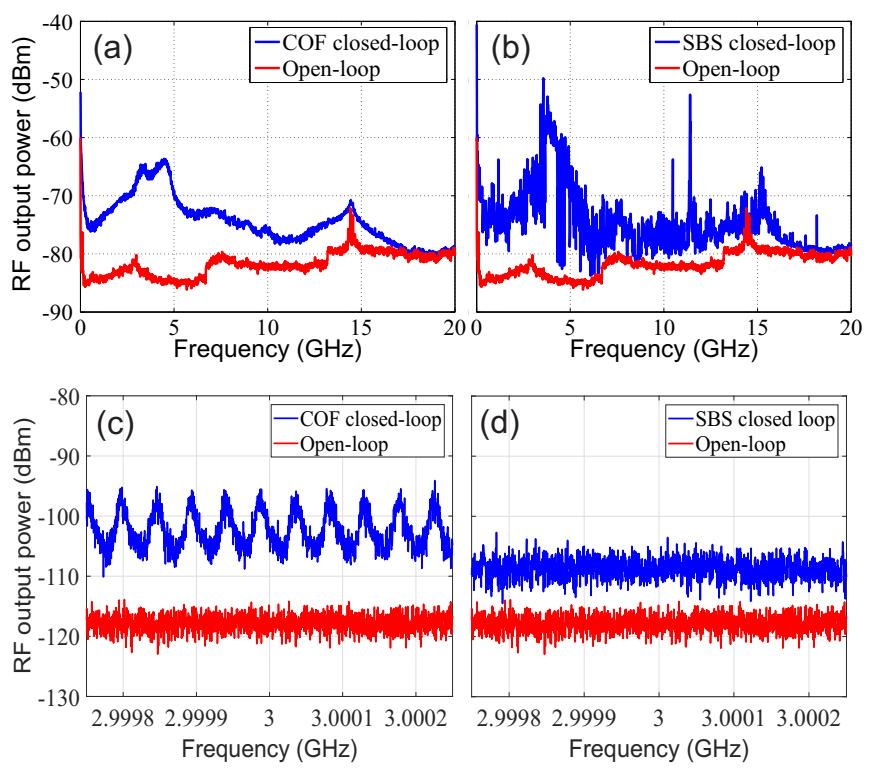

Fig. 4. RF spectra measured at $1000 \mathrm{~mA}$ for (a) the COF closed-loop configuration and (b) SBS closed-loop configuration. For comparison, an RF spectrum for the open-loop configuration is also plotted in each figure. The $y$-axis scale of (b) is the same as the one in (a). (c) and (d) are the zoom-in spectra at a centre-frequency of $3 \mathrm{GHz}$ with a span of $500 \mathrm{kHz}$ for the three configuations. An averaging of 10 has been performed in order to reveal the external-cavity frequency clearly. The y-axis scale of (d) is the same as the one in (c)

RF spectrum mapping is also undertaken by scanning the laser drive current from $0 \mathrm{~mA}$ to $1000 \mathrm{~mA}$ at every $5 \mathrm{~mA}$. Figure 5 presents the RF spectrum maps for COF and SBSOF. The map in the COF configuration with a feedback ratio of $-30 \mathrm{~dB}$ (Fig. 5(a)) manifests very abundant dynamics. In the map, the side-mode is seen at $14.41 \mathrm{GHz}$ and the relaxation oscillation frequency can also be seen as increasing the current. The spectrum in the SBSOF configuration as shown in Fig. 5(b) appears very different to that in the COF one. The relaxation oscillation frequency is clearly visible in the spectrum and this frequency tends toward $2.7 \mathrm{GHz}$ as increasing the laser drive current. In fact, the clear appearance of the relaxation oscillation frequency is attributed to the Ralyleigh scattering from the long fibre. Moreover, the relaxation oscillation frequency starts appearing from $190 \mathrm{~mA}$ whilst SBS starts from $306 \mathrm{~mA}$. So it is found that Rayleigh scattering occurs in the fibre before SBS. In Fig. 5(b), the BFS is clearly seen from $306 \mathrm{~mA}$ at $11.46 \mathrm{GHz}$. As the SBS starts from this current, the peak at $15 \mathrm{GHz}$ and the other at $3.6 \mathrm{GHz}$ also start emerging at the same time. Besides, the internal cavity frequency is shifted from $14.41 \mathrm{GHz}$ to $15 \mathrm{GHz}$ as seen in Fig. 4(b). Despite of the presence of Rayleigh scattering, these peaks are a clear evidence of the strong contribution of SBS. In addition, the SBSOF ratio is $-25 \mathrm{~dB}$ whereas the Rayleigh scattering rate is $-36.7 \mathrm{~dB}$. As SBS is stronger than Rayleigh scattering in the fibre, this noise-like dynamics of the laser mainly originate from SBS. As increasing the current, the spectrum become broader and its power goes higher, leading to stronger power fluctuations. As discussed above, the SBS power saturation is caused by the competition between the strong fluctuation and SBS. So the saturation power of $450 \mu \mathrm{W}$ is a trade-off between them. We have also carried out another mapping in a COF configuration with a feedback ratio of $-36 \mathrm{~dB}$ which is similar to the Rayleigh scattering rate. But in Fig. 5(c) only the relaxation oscillation frequency and the sidemode at $14.41 \mathrm{GHz}$ are observed. Therefore it is confirmed that the complex laser dynamics shown in Fig. 5(b) are strongly attributed to SBS. In the COF configuration, the spectrum can slightly be flattened at $1000 \mathrm{~mA}$ with a strong feedback $(>-20 \mathrm{~dB})$. However, in the SBSOF configuration, we have not observed any flattening of spectrum. It is suggested that this is because of the weak SBS power. Further experiment is needed to investigate this aspect by amplifying the SBSOF.
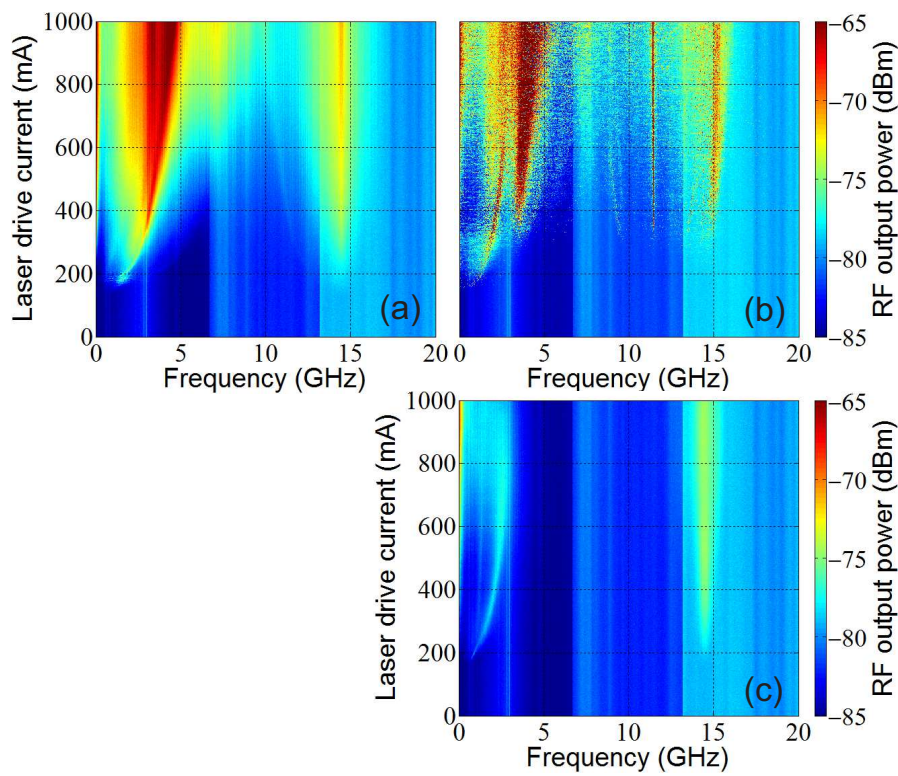

Fig. 5. RF spectrum maps for (a) conventional optical feedback with $-30 \mathrm{~dB}$, (b) stimulated Brillouin scattering optical feedback and (c) conventional optical feedback with $-36 \mathrm{~dB}$. The $\mathrm{y}$-axis scale of (b) is the same as the one in (a) and the colour-coded scale for RF power in (b) is shared with (a).

We have also investigated auto-correlation functions from timetraces for both the configurations. The autocorrelation function is used for analysing dynamical signals and allows revealing any periodicity in the signal. The value of 1 in the ACF means that it is completely correlated at that time shift. Timetraces are recorded at the 10\%-port of the coupler at $1000 \mathrm{~mA}$ by the PD and a $15 \mathrm{GHz}$ digital oscilloscope with 4 
million data points in a window of $100 \mu$ s. So the sampling rate is $40 \mathrm{GS} / \mathrm{s}$ which is enough to pick up any high frequency fluctuation. The autocorrelation function used in this work is defined in Ref. [28] and plotted in Fig. 6. In the figure, the autocorrelation functions for $\mathrm{COF}$ and SBSOF display a very sharp form and they are in fact decorrelated within 1 ns. However, in Fig. 6(a), the autocorrelation function for COF reveals another sharp peak at $21.1 \mu$ s. This peak is the time-delay signature which corresponds to the external cavity frequency of $47 \mathrm{kHz}$. On the other hand, the autocorrelation function for SBSOF in Fig. 6(b) does not manifest such a signature. We have verified that there is no peak neither at around $42.2 \mu \mathrm{s}$ in the SBSOF configuration. Hence, it is evident that SBS contributes to the suppression of the timedelay signature. By comparing to Refs.[25], [26], [27], [28], it is clearly shown from the figure that the TDS is successfully suppressed in our new configuration. It is noticed from the figure that the sidelobe base is slightly thicker than that of Fig. 6(a). This is due to the BFS at $11.46 \mathrm{GHz}$ which can easily be filtered out by a low-pass filter.

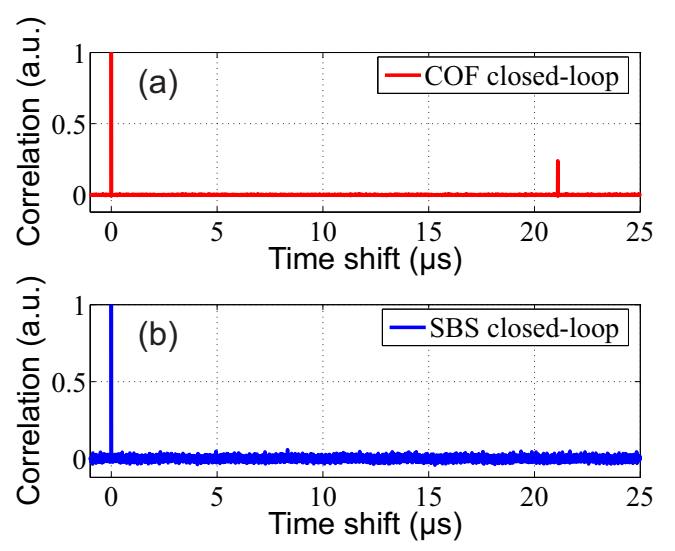

Fig. 6. Autocorrelation functions calculated from a timetrace of 4 million data points over $100 \mu$ s (a) for COF and (b) for SBSOF.

\section{Conclusions}

In conclusion, we have investigated the dynamical behaviour of the laser diode subject to stimulated Brillouin scattering optical feedback (SBSOF). The Brillouin back-scattered light from a $4 \mathrm{~km}$-long fibre is injected into the laser diode for optical feedback. The RF spectrum results clearly indicate the Brillouin frequency shift at $11.46 \mathrm{GHz}$ which is a clear evidence of the presence of stimulated Brillouin scattering (SBS). Such a shift of $11.46 \mathrm{GHz}$ is a very large shift for optical feedback laser diodes. The frequency shift used in frequency-shifted feedback is implemented by an acoustooptic modulator which provides in general a frequency shift of few $100 \mathrm{MHz}$. Hence, the frequency shift of $11.46 \mathrm{GHz}$ is much greater than frequency shifts obtained by acousto-optic modulators. Despite of such a very large frequency shift, the laser diode with SBSOF shows abundant dynamics. Moreover, the SBS rate is around $-25 \mathrm{~dB}$ which is $11.7 \mathrm{~dB}$ stronger than the Rayleigh scattering rate. As such, this is a clear indication that such complex dynamics originate from SBS rather than
Rayleigh scattering. The RF spectrum maps confirm that the optical feedback using SBS strongly contributes to complex dynamical behaviours of the laser diode. By comparing qualitatively with the one for conventional optical feedback (COF), it is clearly seen that the spectral aspect is different for both the feedback configuration. In the SBSOF configuration, the time-delay signature is also successfully suppressed as seen in the autocorrelation function investigation unlike that in the COF configuration.

As Brillouin scattering is described as a random process, SBS provides random feedback which also allows generating complex dynamical behaviours. In the fundamental point of view, this is the first study showing complex dynamical behaviours in a laser diode subject to random feedback via SBS. The signals obtained from random SBSOF are expected to be stochastic. Further study is being carried out to determine the randomness of the signals by studying Lyapunov exponents and Kolmogorov entropy.

In the engineering application point of view, our new random feedback scheme which allows vanishing the time-delay signature is promising in the engineering applications such as enhancing the randomness in random number generation or the security in secure chaos communications. Compared to the other works which uses post-signal processing[25], [26], [27], [28], our configuration is the first configuration of random feedback which allows generating complex signals directly without the presence of the time-delay signature, not suppressing it by any post-signal processing. It can also be used for sensing application as in sensing application with chaotic lasers.

\section{ACKNOWLEDGEMENTS}

One of the authors, A. G. Correa-Mena wishes to thank the Mexican Consejo Nacional de Ciencia y Tecnología (CONACyT) for the student scholarship (Reference no. 335148) and supporting her research stay in France.

\section{REFERENCES}

[1] R. Lang and K. Kobayashi, "External optical feedback effects on semiconductor injection laser properties," IEEE J. Quantum Electron., vol. 16 , no. 3, pp. 347-355, Mar. 1980.

[2] J. Mork, B. Tromborg, and J. Mark, "Chaos in semiconductor lasers with optical feedback: theory and experiment," IEEE J. Quantum Electron., vol. 28, no. 1, pp. 93-108, Jan. 1992.

[3] R. Tkach and A. Chraplyvy, "Regimes of feedback effects in 1.5-\#181;m distributed feedback lasers," J. Lightwave Technol., vol. 4, no. 11, pp. 1655-1661, Nov. 1986.

[4] J. Ohtsubo, "Chaos synchronization and chaotic signal masking in semiconductor lasers with optical feedback," IEEE J. Quantum Electron., vol. 38, no. 9, pp. 1141-1154, Sep. 2002.

[5] M. Sciamanna and K. A. Shore, "Physics and applications of laser diode chaos," Nat. Photonics, vol. 9, no. 3, pp. 151-162, Mar. 2015. [Online]. Available: https://www.nature.com/articles/nphoton.2014.326

[6] C. Otto, K. Lüdge, and E. Schöll, "Modeling quantum dot lasers with optical feedback: sensitivity of bifurcation scenarios," Phys. Status Solidi (b), vol. 247, no. 4, pp. 829-845, 2010. [Online]. Available: https://onlinelibrary.wiley.com/doi/abs/10.1002/pssb.200945434

[7] F. Baladi, M. W. Lee, J.-R. Burie, M. A. Bettiati, A. Boudrioua, and A. P. A. Fischer, "High-resolution lowfrequency fluctuation map of a multimode laser diode subject to filtered optical feedback via a fiber Bragg grating," Opt. Lett., vol. 41, no. 13, pp. 2950-2953, Jul. 2016. [Online]. Available: https://www.osapublishing.org/ol/abstract.cfm?uri=ol-41-13-2950 
[8] E. Mercier, D. Wolfersberger, and M. Sciamanna, "Highfrequency chaotic dynamics enabled by optical phase-conjugation," Sci. Rep., vol. 6, p. 18988, Jan. 2016. [Online]. Available: https://www.nature.com/articles/srep18988

[9] D. Rontani, E. Mercier, D. Wolfersberger, and M. Sciamanna, "Enhanced complexity of optical chaos in a laser diode with phase-conjugate feedback," Opt. Lett., vol. 41, no. 20, pp. 4637-4640, Oct. 2016. [Online]. Available: https://www.osapublishing.org/ol/abstract.cfm?uri=ol-41-204637

[10] J. Paul, M. W. Lee, and K. A. Shore, "Effect of chaos pass filtering on message decoding quality using chaotic external-cavity laser diodes,' Opt. Lett., vol. 29, no. 21, pp. 2497-2499, Nov. 2004. [Online]. Available: https://www.osapublishing.org/ol/abstract.cfm?uri=ol-29-212497

[11] A. Argyris, D. Syvridis, L. Larger, V. Annovazzi-Lodi, P. Colet, I. Fischer, J. García-Ojalvo, C. R. Mirasso, L. Pesquera, and K. A Shore, "Chaos-based communications at high bit rates using commercial fibre-optic links," Nature, vol. 438, no. 7066, pp. 343-346, Nov. 2005. [Online]. Available: https://www.nature.com/articles/nature04275

[12] C. Xue, N. Jiang, Y. Lv, C. Wang, G. Li, S. Lin, and K. Qiu, "Security-enhanced chaos communication with timedelay signature suppression and phase encryption," Opt. Lett., vol. 41, no. 16, pp. 3690-3693, Aug. 2016. [Online]. Available: https://www.osapublishing.org/ol/abstract.cfm?uri=ol-41-16-3690

[13] L. Wang, Y. Guo, P. Li, T. Zhao, Y. Wang, and A. Wang, "White-Chaos Radar With Enhanced Range Resolution and Anti-Jamming Capability," IEEE Photon. Technol. Lett., vol. 29, no. 20, pp. 1723-1726, Oct. 2017.

[14] L. Xia, D. Huang, J. Xu, and D. Liu, "Simultaneous and precise fault locating in WDM-PON by the generation of optical wideband chaos," Opt. Lett., vol. 38, no. 19, pp. 3762-3764, Oct. 2013. [Online]. Available: https://www.osapublishing.org/ol/abstract.cfm?uri=ol-38-193762

[15] A. Uchida, K. Amano, M. Inoue, K. Hirano, S. Naito, H. Someya, I. Oowada, T. Kurashige, M. Shiki, S. Yoshimori, K. Yoshimura, and P. Davis, "Fast physical random bit generation with chaotic semiconductor lasers," Nat. Photonics, vol. 2, no. 12, pp. 728-732, Dec. 2008. [Online]. Available: https://www.nature.com/articles/nphoton.2008.227

[16] L. Larger, M. C. Soriano, D. Brunner, L. Appeltant, J. M. Gutierrez, L. Pesquera, C. R. Mirasso, and I. Fischer, "Photonic information processing beyond Turing: an optoelectronic implementation of reservoir computing," Opt. Express, vol. 20, no. 3, pp. 3241-3249, Jan. 2012. [Online]. Available: https://www.osapublishing.org/oe/abstract.cfm?uri=oe-20-3-3241

[17] Y. Kuriki, J. Nakayama, K. Takano, and A. Uchida, "Impact of input mask signals on delay-based photonic reservoir computing with semiconductor lasers," Opt. Express, vol. 26, no. 5, pp. 5777-5788, Mar. 2018. [Online]. Available: https://www.osapublishing.org/oe/abstract.cfm?uri=oe-26-5-5777

[18] J. A. Reinoso, J. Zamora-Munt, and C. Masoller, "Extreme intensity pulses in a semiconductor laser with a short external cavity," Phys. Rev. E, vol. 87, no. 6, p. 062913, Jun. 2013. [Online]. Available: https://link.aps.org/doi/10.1103/PhysRevE.87.062913

[19] M. W. Lee, F. Baladi, J.-R. Burie, M. A. Bettiati, A. Boudrioua and A. P. A. Fischer, "Demonstration of optical rogue waves using a laser diode emitting at $980 \mathrm{~nm}$ and a fiber Bragg grating," Opt. Lett., vol. 41, no. 19, pp. 4476-4479, Oct. 2016. [Online]. Available: https://www.osapublishing.org/ol/abstract.cfm?uri=ol-41-19-4476

[20] X. Fu, S.-C. Chan, Q. Liu, and K. K.-Y. Wong, "Broadband optical chaos for stimulated Brillouin scattering suppression in power over fiber," Appl Opt, vol. 50, no. 25, pp. E92-E96, Sep. 2011. [Online]. Available: https://www.osapublishing.org/ao/abstract.cfm?uri=ao-50-25-E92

[21] J. Zhang, M. Zhang, M. Zhang, Y. Liu, C. Feng, Y. Wang, and Y. Wang, "Chaotic Brillouin optical correlation-domain analysis," Opt. Lett., vol. 43, no. 8, pp. 1722-1725, Apr. 2018. [Online]. Available: https://www.osapublishing.org/ol/abstract.cfm?uri=ol-43-8-1722

[22] M. Santagiustina and L. Ursini, "Dynamic Brillouin gratings permanently sustained by chaotic lasers," Opt. Lett., vol. 37, no. 5, pp. 893-895, Mar. 2012. [Online]. Available: https://www.osapublishing.org/ol/abstract.cfm?uri=ol-37-5-893

[23] J. Zhang, Z. Li, Y. Wu, M. Zhang, Y. Liu, and M. Li, "Optimized chaotic Brillouin dynamic grating with filtered optical feedback," Sci. Rep., vol. 8, no. 1, p. 827, Jan. 2018. [Online]. Available: https://www.nature.com/articles/s41598-018-19180-w

[24] G. Agrawal, Nonlinear Fiber Optics, A. Press, Ed Academic Press, 2006, no. pp. 355-388. [Online]. Available: http://linkinghub.elsevier.com/retrieve/pii/B9780123695161X50006
[25] A. Wang, Y. Yang, B. Wang, B. Zhang, L. Li, and Y. Wang, "Generation of wideband chaos with suppressed timedelay signature by delayed self-interference," Optics Express, vol. 21, no. 7, pp. 8701-8710, Apr. 2013. [Online]. Available: https://www.osapublishing.org/oe/abstract.cfm?uri=oe-21-7-8701

[26] J. Zhang, C. Feng, M. Zhang, Y. Liu, and Y. Zhang, "Suppression of Time Delay Signature Based on Brillouin Backscattering of Chaotic Laser," IEEE Photon. J., vol. 9, no. 2, pp. 1-8, Apr. 2017.

[27] J. Zhang, M. Li, A. Wang, M. Zhang, Y. Ji, and Y. Wang, "Time-delay-signature-suppressed broadband chaos generated by scattering feedback and optical injection," Applied Optics, vol. 57, no. 22, pp. 6314-6317, Aug. 2018. [Online]. Available: https://www.osapublishing.org/ao/abstract.cfm?uri=ao-57-22-6314

[28] N. Jiang, C. Wang, C. Xue, G. Li, S. Lin, and K. Qiu, "Generation of flat wideband chaos with suppressed time delay signature by using optical time lens," Opt. Express, vol. 25, no. 13, pp. 14359-14367, Jun. 2017. [Online]. Available: https://www.osapublishing.org/oe/abstract.cfm?uri=oe-25-13-14359

[29] K. A. Shore and D. M. Kane, "Comb generation bandwidth for frequency-shifted feedback semiconductor lasers," IEEE J. Quantum Electron., vol. 35, no. 7, pp. 1053-1056, Jul. 1999.

[30] Y. Noblet, J. P. Toomey, and D. M. Kane, "Dynamics of a semiconductor laser with frequency shifted feedback," in Proc. SPIE 8980, Physics and Simulation of Optoelectronic Devices XXII, vol. 8980. International Society for Optics and Photonics, Mar. 2014, p. 89800C. [Online]. Available: https://www.spiedigitallibrary.org/conference-proceedings-ofspie/8980/89800C/Dynamics-of-a-semiconductor-laser-with-frequencyshifted-feedback/10.1117/12.2038871.short 